\title{
Oocyte-like cells induced from mouse spermatogonial stem cells
}

\author{
Lu Wang ${ }^{1}$, Jinping Cao ${ }^{2}$, Ping Ji ${ }^{1}$, Di Zhang ${ }^{1}$, Lianghong $\mathrm{Ma}^{3}$, Martin Dym ${ }^{4}$, Zhuo $\mathrm{Yu}^{1 *}$ and Lixin Feng ${ }^{1,2,4^{*}}$
}

\begin{abstract}
Background: During normal development primordial germ cells (PGCS) derived from the epiblast are the precursors of spermatogonia and oogonia. In culture, PGCs can be induced to dedifferentiate to pluripotent embryonic germ (EG) cells in the presence of various growth factors. Several recent studies have now demonstrated that spermatogonial stem cells (SSCS) can also revert back to pluripotency as embryonic stem (ES)-like cells under certain culture conditions. However, the potential dedifferentiation of SSCs into PGCs or the potential generation of oocytes from SSCs has not been demonstrated before.

Results: We report that mouse male SSCs can be converted into oocyte-like cells in culture. These SSCs-derived oocytes (SSC-OoCs) were similar in size to normal mouse mature oocytes. They expressed oocyte-specific markers and gave rise to embryos through parthenogenesis. Interestingly, the $Y$ - and $X$-linked testis-specific genes in these SSC-Oocs were significantly down-regulated or turned off, while oocyte-specific $X$-linked genes were activated. The gene expression profile appeared to switch to that of the oocyte across the $X$ chromosome. Furthermore, these oocyte-like cells lost paternal imprinting but acquired maternal imprinting.

Conclusions: Our data demonstrate that SSCs might maintain the potential to be reprogrammed into oocytes with corresponding epigenetic reversals. This study provides not only further evidence for the remarkable plasticity of SSCs but also a potential system for dissecting molecular and epigenetic regulations in germ cell fate determination and imprinting establishment during gametogenesis.
\end{abstract}

Keywords: Gametogenesis, Oocyte, PGC, Sex reversal, Spermatogonial stem cells

\section{Background}

Despite the different genotypes of germ cells in males with XY cells and females with XX cells, both types of germ cells share the same progenitors, namely, primordial germ cells (PGCs). The differentiation of PGCs into either the male or female phenotype takes place in the sex glands at later stages of embryonic development, and sexual differentiation of the germ cells is controlled by the somatic environment of the gonad rather than the sex chromosome constitution of the germ cells themselves [1-3]. Somatic mutation of sex-determining genes contributes to the sex reversal of XY germ cells to oogonia during gonad development; thus, the fate of $\mathrm{XY}$

\footnotetext{
* Correspondence: yuzhuo78@shsmu.edu.cn; fenglx@shsmu.edu.cn 'Institute of Medical Sciences, Shanghai JiaoTong University School of Medicine, 280 Chongqing S. Road, Shanghai 200025, China

${ }^{2}$ Institute of Health Sciences, Shanghai Institutes for Biological Sciences, Chinese Academy of Sciences, Shanghai, China

Full list of author information is available at the end of the article
}

male germ cells varies in response to environmental signaling in the gonad [4]. A few recent studies have demonstrated that spermatogonial stem cells (SSCs), which are the progeny of PGCs/gonocytes, can be reprogrammed into embryonic stem-like cells in vitro without transgene manipulation [5-9], indicating that SSCs retain remarkable plasticity. In addition, XY embryonic stem cells (ESCs) can differentiate into oocytes in culture [10]. Therefore, it is interesting to know whether SSCs can be reprogrammed into female germ cells. Here, we report that SSCs can be converted into oocyte-like cells in culture.

\section{Results}

Oocyte-like cells derived from SSCs in culture

We started with SSCs isolated by magnetic-activated cell sorting (MACS) with a GFRa1 [11] antibody and obtained GFRa1 $^{(+)}$SSCs [12] (Figure 1A) from 8-day old OG2 transgenic mice (C57/B6 transgenic mice carrying

\section{Biomed Central}




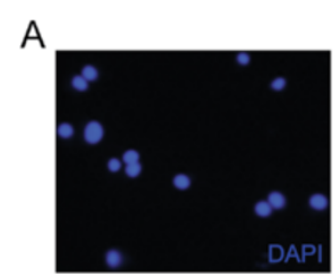

B

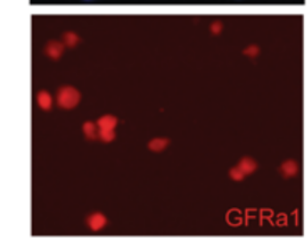

D
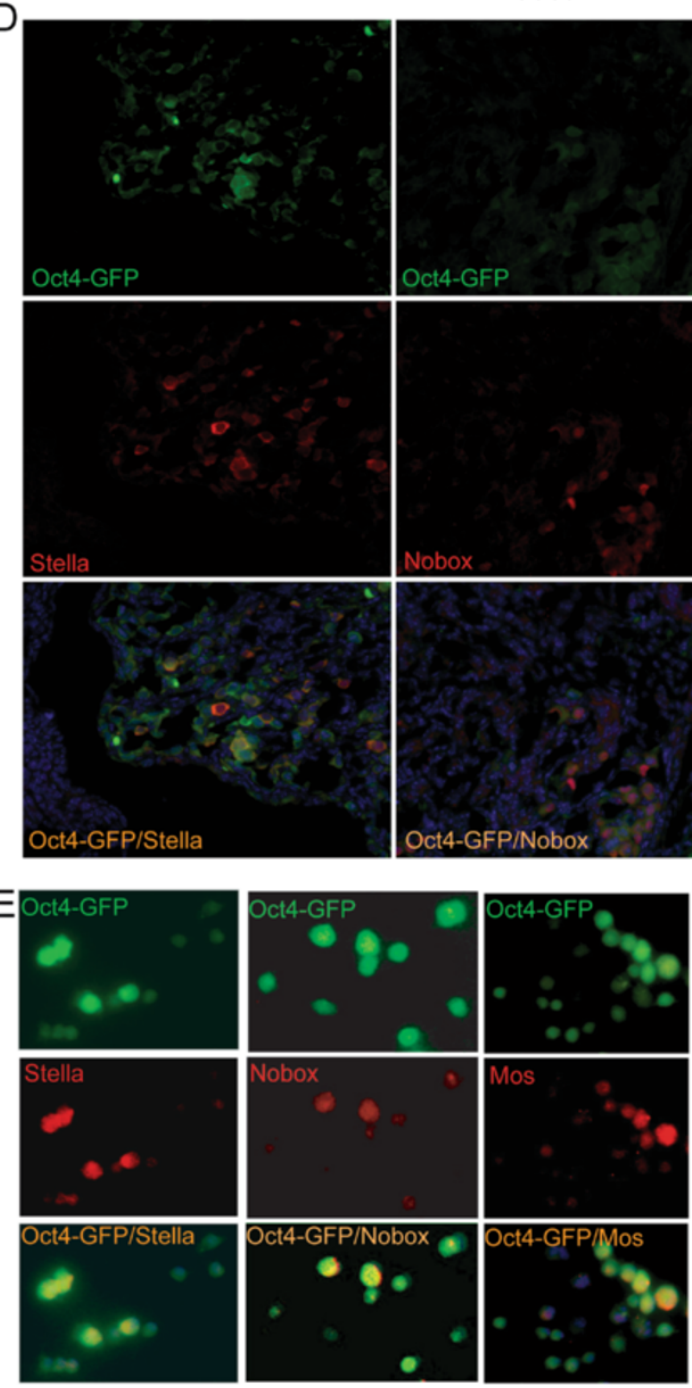

SSCs
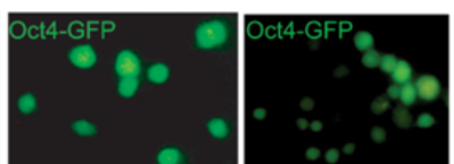

C
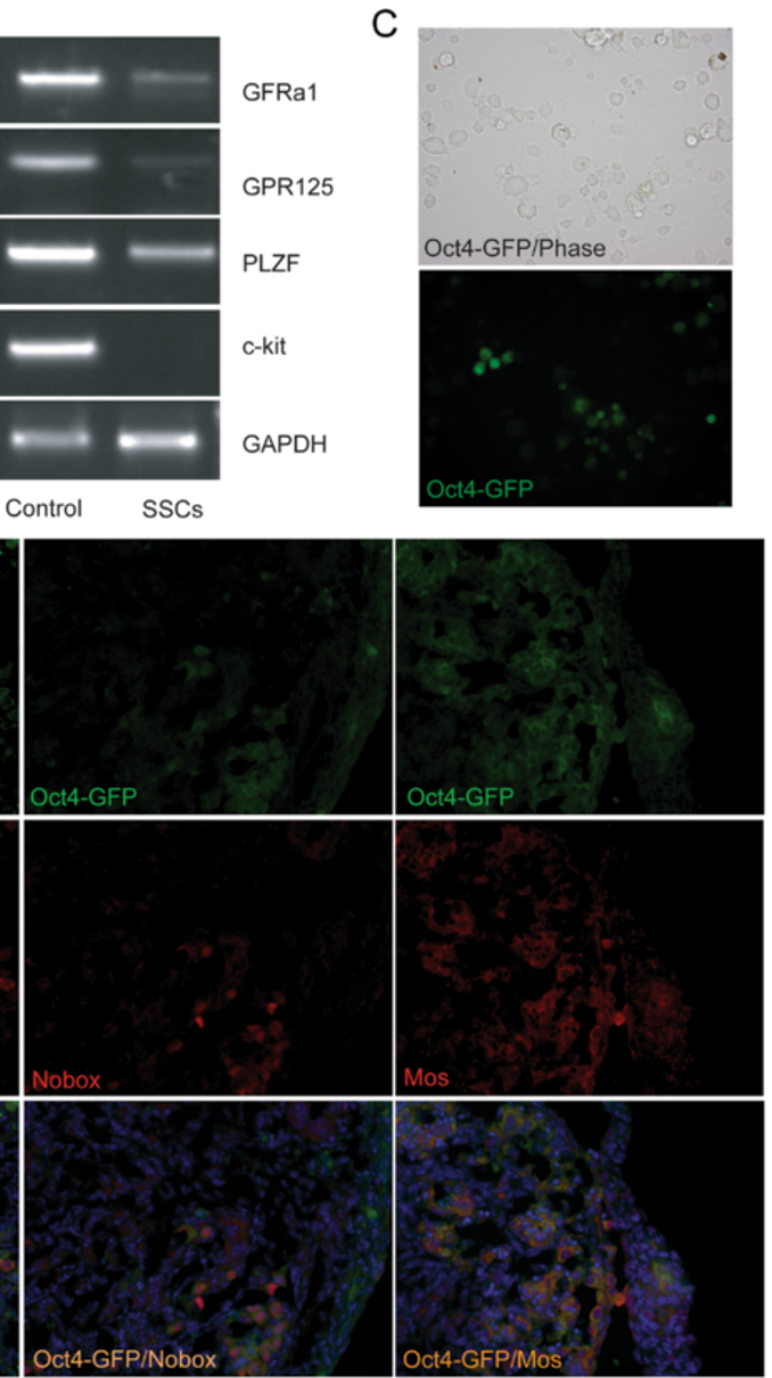

F

口oct4 ${ }^{+}$cells

$\square$ Nobox $^{+} / \mathrm{Mos}^{+} /$Stella $^{+}$cells

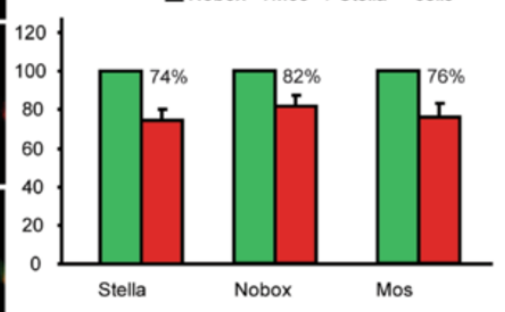

Figure 1 (See legend on next page.) 


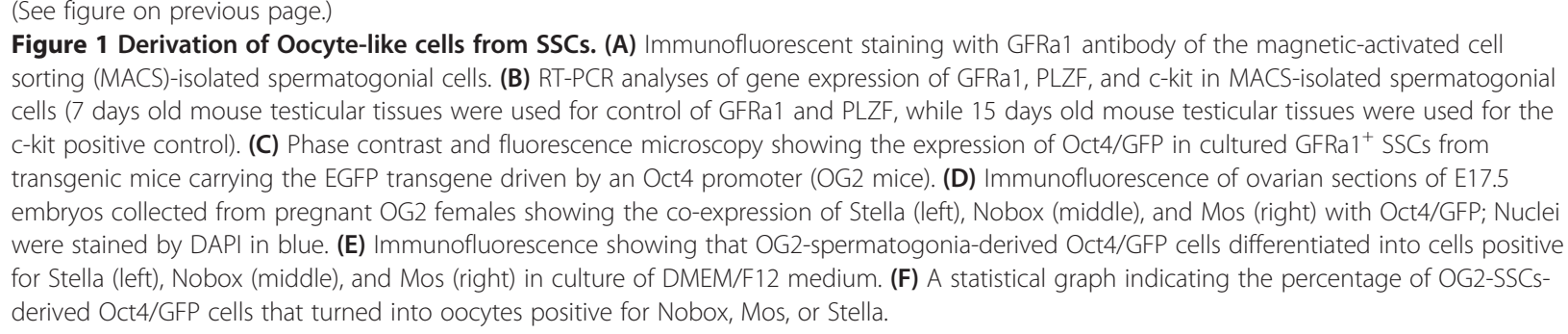

the EGFP transgene driven by an Oct4 promoter). The isolated SSCs were further characterized by RT-PCR analyses for the positive and negative markers of SSCs (Figure 1B). We then cultured them in KO-DMEM medium containing $1 \%$ fetal bovine serum (FBS), 1,500 units/ml leukemia inhibitory factor (LIF) and $2 \mathrm{i}(2 \mu \mathrm{M}$ SU5402 plus $3 \mu \mathrm{M}$ CHIR99021) for one week, which synergize with the LIF signaling in pluripotency reprogramming $[13,14]$. Within the first week of culture, $20 \%$ the Oct4/GFP expressing cells appeared (Figure 1C), indicating the dedifferentiation of SSCs under this culture condition. Our preliminary study demonstrated that DMEM/F12 medium supplemented with 15\% FBS and LIF plus follicle-stimulating hormone (FSH), Epidermal growth factor (EGF), B27, and Insulin-Transferrin-Selenium-A (ITS) was useful in growing germ cell nuclear antigen( GCNA1)-positive germ cells from adult ovarian cells (Additional file 1: Figure S1A). Thus, we used this culture condition to test whether oogonial fate from the GFPexpressing cells can be induced. Under this culture condition for one more week, most of the GFP-expressing cells grew larger than SSCs. Interestingly, RT-PCR analyses indicated that oocyte-specific genes, including GDF-9 [15], Nobox [16], and Oogenesin [17], were expressed in the large cells (Additional file 1: Figure S1B). Thus, the oocyte-like cells appear to be derived from SSCs in culture. Furthermore, we used ovarian sections from E17.5 OG2 embryos as a control (Figure 1D) for the staining of Nobox, c-Mos, and Stella to examine the development of oocytes from GFP-positive cells (Figure 1E). We found that Oct4/GFP positive cells turned into Noboxexpressing cells (82\%), Mos-expressing cells (76\%), and Stella-expressing cells (74\%) (Figure 1F). More interestingly, with extended culture by day 21, the SSC-derived oocytes (SSC-Oocs) grew larger (Figure $2 \mathrm{~A}$ ) and $\sim 60 \%$ of these cells became oocytes resembling that of germinal vesicle $(\mathrm{GV})$ stage (Figure $2 \mathrm{~B})$. They were further demonstrated by the formation of the 'surrounded nucleolus' (Figure 2C and D), a typical chromatin configuration in mouse oocyte at GV stage [18]. Among these growing oocytes, $10 \%$ grew to size similar to mature oocytes from mice (Figure 2E). Oocyte-specific markers, including H1Foo [19], zonapellucida 3 (ZP3), GDF-9, and SCP3 were expressed in these cells as demonstrated by RT-PCR analysis (Figure 3A). Meiotic and haploid SSC-Oocs were also identified by SCP3 staining and Giemsa stainingrespectively (Additional file 2: Figure 2). More surprisingly, $\sim 2 \%$ SSC-Oocs with a structure like a polar body were also generated in culture (Figure $2 \mathrm{~F}$ and $\mathrm{G}$ ), and even gave rise to embryos (Figure 2J and $\mathrm{K}$ ), most likely via parthenogenesis. The formation of embryos was further confirmed by the expression of genes from preimplantation embryos, such as Hmgpi and Trim43a [20,21] (Figure 3B). To test whether the SSC-Oocs were capable of being fertilized by sperm, we generated oocytes from $\mathrm{BABL} / \mathrm{c}$ male pups using the same approach and used sperm from OG2 mice to carry out intracytoplasmic sperm injection (ICSI). In our early attempts, all SSC-Oocs died shortly after injection; The SSC-Oocs were very fragile, and they were severely damaged while being picked up and injected. To avoid this problem, we performed ICSI in the original culture dish without picking up SSC-Oocs before injection. We achieved success in fertilizing SSC-Oocs and obtained 4-cell embryos expressing GFP, which was carried by the sperm from OG2 mice (Figure $2 \mathrm{H}$ and I). Overall, with 53 attempts of ICSI, we obtained 5 embryos of early developmental stages after artificial activation with none beyond 4-cells. Collectively, the conditions for the oocyte induction from SSCs is summarized in Figure 2L.

\section{Primordial germ cells might be the intermediates from SSCs to Oocytes}

To further confirm the genotype of these SSC-Oocs, we carried out a PCR analysis of Sry, which is located only on the $\mathrm{Y}$ chromosome, in the GFP-expressing larger SSC-Oocytes. We found that Sry was not present (Figure $3 \mathrm{C}$ ), indicating that these SSC-oocytes should be of the XO karyotype and that YO cells died while growing due to the lack of whole X-linked genes.

Because ESCs can develop into oocytes [10] while cultured SSCs can be reverted to pluripotent cells [5-9], it has been proposed that cultured SSCs may be converted to pluripotent cells that subsequently develop into oocytes. A second hypothesis is that the cultured SSCs first revert to PGCs, which then develop into the oocytes. Moreover, the cultured SSCs can directly transdifferentiate into oocytes. To understand the potential mechanisms underlying the conversion of SSCs into 

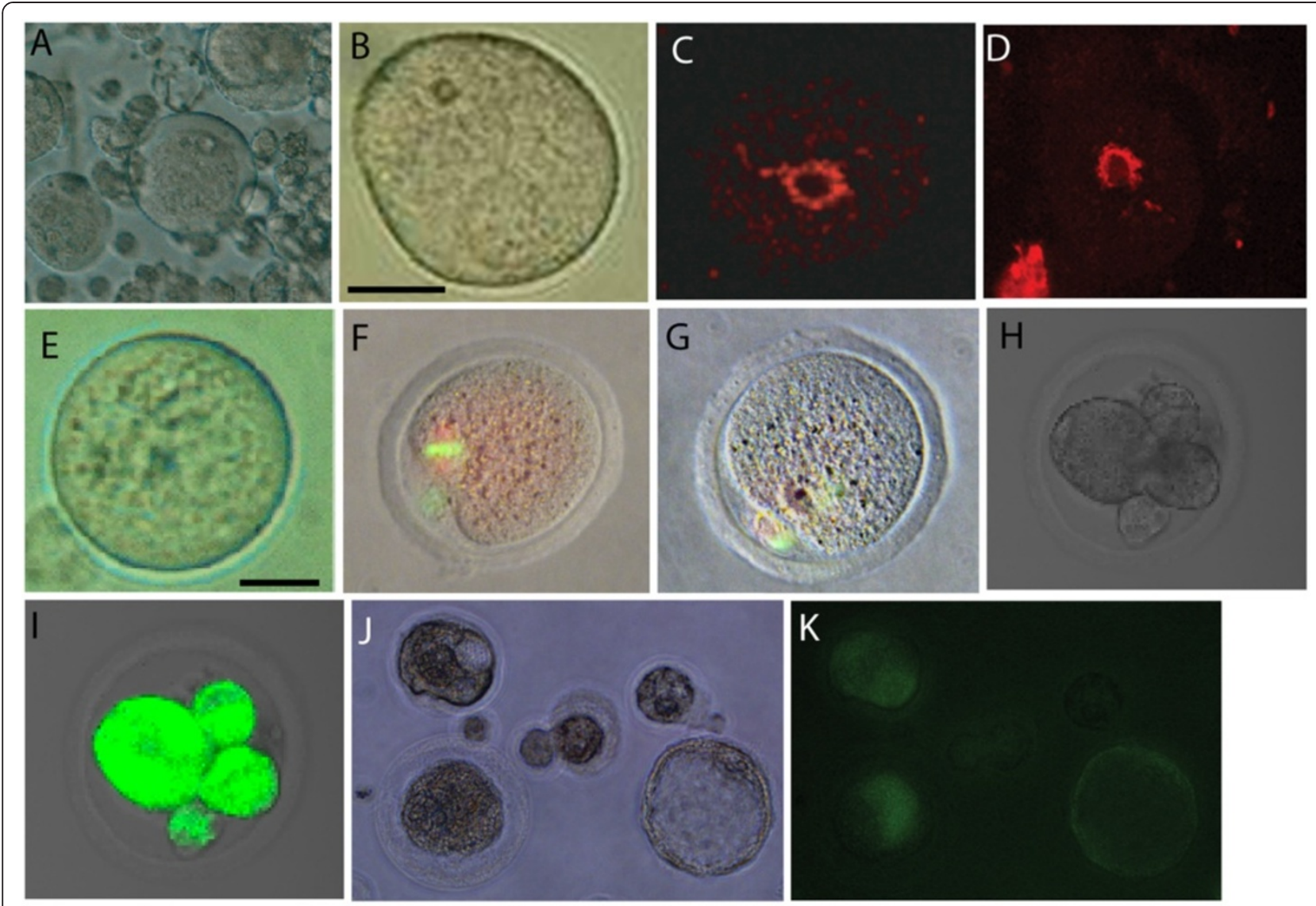
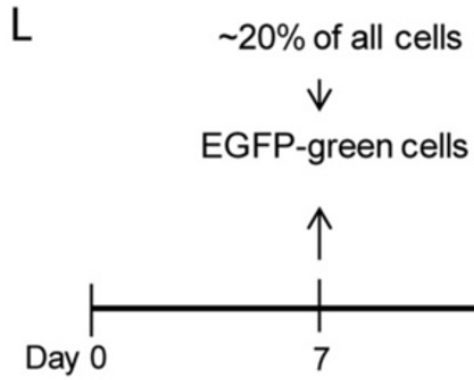

A $\sim 60 \%$ of EGFP green cells $\downarrow$ Large cells resembling GV oocytes

\section{$\sim 2 \%$ of large cells}

Mature oocye-like cells with a polar body

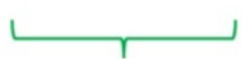

\section{KO-DMEM 1\%FBS+LIF+2i}

\section{$\mathrm{DMEM} / \mathrm{F} 12+15 \% \mathrm{FBS}+\mathrm{LIF}+\mathrm{FSH}+\mathrm{EGF}+\mathrm{B} 27+\mathrm{ITS}$}

Figure 2 Characterization of mature oocytes and embryos derived from spermatogonial stem cells (SSCs) in culture. (A and B) Phase contrast microscopy of growing oocytes from the culture of SSCs (A), a SSCs-derived oocyte (SSC-Ooc) resembling that of germinal vesicle (GV) stage (B,scale bar $=30 \mu \mathrm{m}$ ). (C and D) Hoechst staining of a GV oocyte from a mouse ovary (C) and OG2-SSC-Ooc (D) showing a rim of chromatin around the nucleolus (the surrounded nucleolus). (E) Phase contrast microscopy of a fully-grown SSC-Ooc, scale bar $=30 \mu \mathrm{m}$. (F and G) YoYo1(green) and lamin B1 (red) staining of a control MII oocyte from a normal mouse ovary (F) and an oocyte with a polar body derived from SSC (G). (H and I) A 4-cell embryo was generated from SSC-oocyte by ICSI with sperm from an OG2 mouse, from which GFP gene was carried by sperm and was expressed in the resulting embryo (I). ( $\mathbf{J}$ and $\mathbf{K}$ ) Phase contrast microscopy of parthenogenetic embryos developed from OG2SSC-oocytes in culture, Oct4/GFP from OG2 strain was expressed in the embryos (K). (L) Schematic representation of the reprogramming conditions from SSCs to oocyte-like cells. 
A

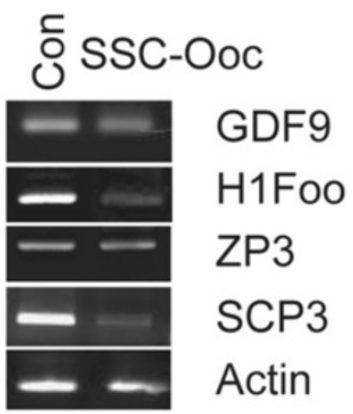

C
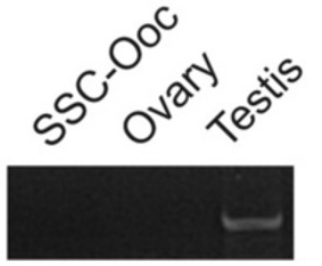

Sry

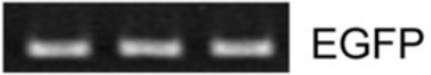

B

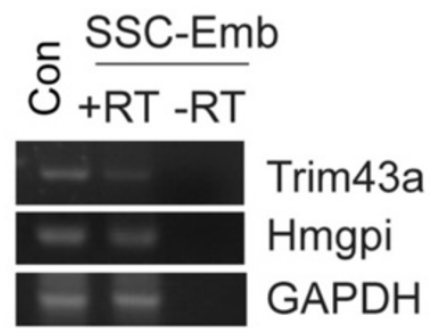

D

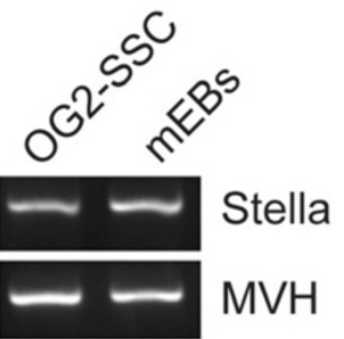

GAPDH

\section{E कृ \\ ণ্

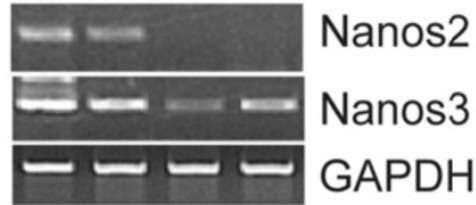

Figure 3 Gene expression analysis of SSCs-derived oocyte (SSC-Oocs) and embryos derived from SSC-Oocs. (A) RT-PCR analyses of the expression of GDF9, H1Foo, ZP3, and SCP3 in OG2-SSC-Oocs (Ovarian RNAs from a 30-day-old female were used as the control; 150 SSC-Oocs of day 21 were collected for RT-PCR). (B) RT-PCR analyses showing the expression of Trim43a and Hmgpi in SSCs-derived embryos (embryos of E3.0 were collected from pregnant mice as a control; SSC-Emb, parthenogenetic embryos developed from SSC-Oocs). (C) PCR analysis of Sry gene in the genomic DNAs from mouse testis, ovary, and OG2-SSC-Oocs, EGFP was used as an internal control for all three genomic DNA samples prepared from OG2 mice. (D) RT-PCR analyses of the expression of Stella and MVH in the OG2-SSCs cultured for one week; mEBs (mouse embryoid bodies) were used as controls. (E) RT-PCR analyses of the expression of Nanos2 and Nanos3 in 5 days of culture of OG2-SSCs, mouse gonad tissue was used as a control.

oocyte-like cells, we examined the expression of genes related to PGC development in the early cultured SSCs. Interestingly, RT-PCR analyses showed that early cultured SSCs expressed PGC development-related genes, including Stella (Dppa3) [22], Vasa $(M V H)$ [23], and Nanos3 [24] (Figure 3D and E). Furthermore, we carried out a time-course staining cultured SSCs from BALB/c mice for PGC markers, including Nanos2, Nanos3, Nanog [25], and Blimp1 [26], and for the oocyte marker Nobox. Nanos2 [27] expression was lost within 3 days, while Nanos3 was maintained in more than $85 \%$ cells through two weeks of culture (Figure 4A and S3); the PGC markers, including Nanog and Blimp1, were induced around day 3 (Figure 4A and Additional file 3: Figure S3). Notably, Nanog expression was gradually lost before day 7, while Nobox expression started between day 5 and day 7 (Figure 4A and Additional file 1: Figure S3). We further confirmed the presence of oocytes by co-staining of multiple markers of oocytes. As shown in Figures 4B-D, Blimp1 positive cells expressed Nobox and Stella, while GDF9 positive cells expressed Nobox. Moreover, compact colonies of embryonic stem-like cells were never observed in our cultures. In addition, E-cadherin, which is highly expressed in mouse ESCs,was not detectable in these cells, and teratoma could not form from them (data not shown). These 


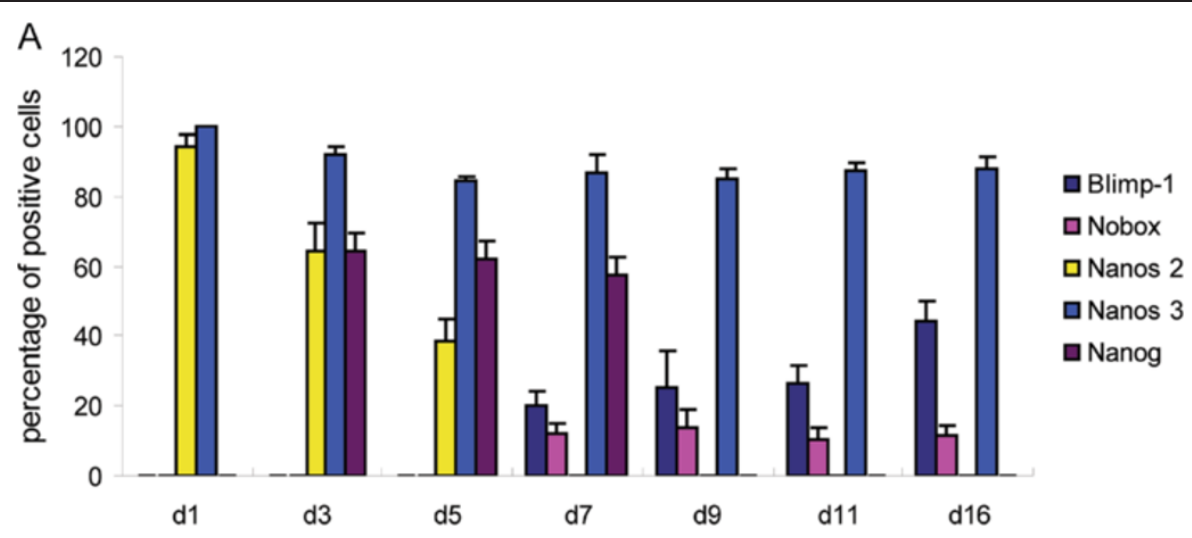

\section{B}
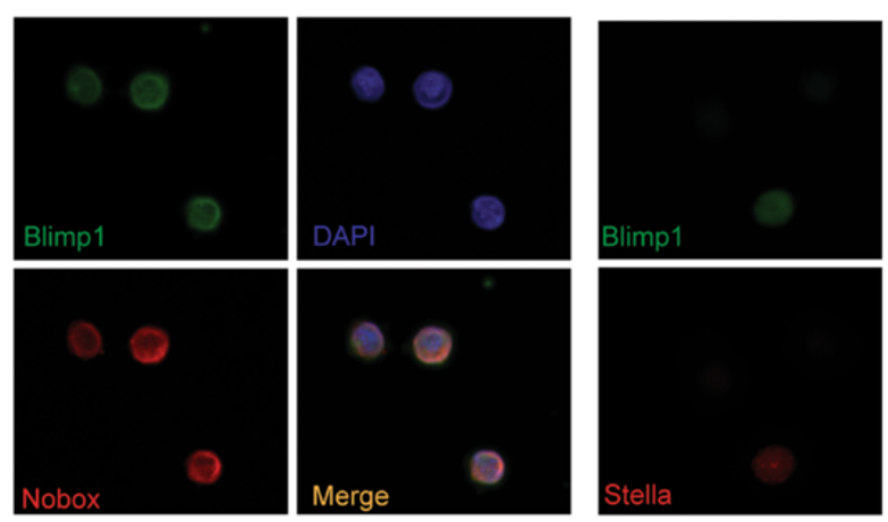

C

D
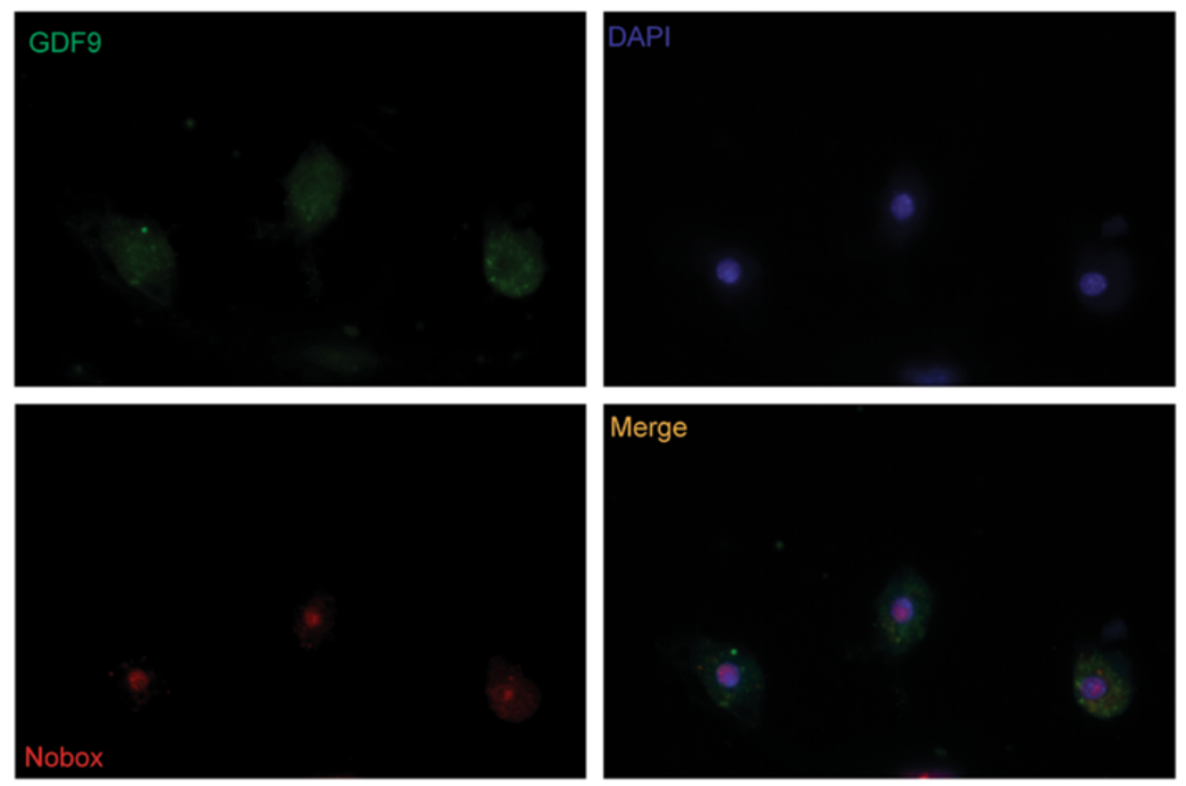

Figure 4 Examination of the markers of PGCs and oocytes by immunofluorescent staining. (A) Time course immunofluorescence analysis of the expression of Nanos2, Nanos3, Nanog, Blimp1, and Nobox, the percentage of positive cells for each corresponding marker at different days of culture was statistized. Data are means \pm SE. $(n=4)$. (B-D) Co- immunofluorescence of Blimp1/Nobox (B), Blimp1/Stella (C), and GDF9/Nobox (D) in SSCs-derived oocyte. 
results indicate that ESC-like cells were less likely to form in this culture but PGCs and oocytes were produced. Therefore, the cultured SSCs probably developed into oocytes through PGC intermediates.

\section{Sex-specific imprint pattern and sex chromosome-linked gene activation are reversed during the conversion SSCs into oocytes}

Parental imprints are established during gametogenesis and are essential for the function of gametes and the normal development of embryos. Thus, we were interesting to learn if imprinting reversals can be induced during the conversion of SSCs into oocytes. We examined the maternally-expressed imprinting gene $p 57 K I P 2$ [28] in the OG2-SSC-Oocs by immunofluorescence and found that $p 57$ KIP2 was expressed in Oct4/GFP positive large cells (Figure 5A). Furthermore, we employed bisulfite genomic sequencing to examine the methylation status of the H19-, Snrpn-, and Dik-Gtl2/meg3-imprinting control regions in SSC-Oocs. We found that the DMR of maternally imprinted Snrpn was methylated, while the DMR of paternally imprinted Igf-H19 and Dik-Gtl2/ meg3 were highly unmethylated, in comparison with freshly isolated SSCs from 8-day old mouse testes (Figure 5B). These results indicate that the epigenetic switching of imprints was associated with the transdifferentiation of SSCs into oocytes.

The activity of X chromosome-linked genes in male germ cells is different from that of female germ cells. The Y chromosome genes have been reported to be essential for spermatogenesis but not for oogenesis. Therefore, we addressed the gene activity status of sex chromosomes in the SSC-Oocs. We examined the expression of sex-dependent X- and Y-linked genes [29,30] and found that the $\mathrm{X}$-linked testis specific genes were significantly down-regulated or turned off (Figure 5C and Additional file 4: Table S2), while oocyte specific genes including GDF9, X-linked BMP15 and Usp $9 x$ were turned on (Figure 5D). The Y-linked genes were silenced (Figure 5C and Additional file 4: Table S2). The loss of the expression of Y-linked gene could be the result of the absence of the Y chromosome in the SSC-Oocytes since YO cells might die. These data indicate that the gene expression pattern of the $\mathrm{X}$ chromosome was changed in favor of the formation of oocytes from SSCs. Thus, following the process of SSCs dedifferentiation back to gonocytes/PGCs and transdifferentiation into oocytes, the $\mathrm{X}$ chromosome might be subjected to large scale changes in gene expression and epigenetic modifications. This hypothesis was supported by the reversal expression of Usp $9 x$ (Figure 5D and Additional file 2: Table S2), which is X-linked and expressed in both male and female embryonic germ cells, but turned off in male germ cells after birth [31]. Therefore, along with the morphological changes during the conversion of SSCs into oocytes, the epigenetic network was converted into the female germ cell form.

\section{Discussion}

In mice, cellular pluripotency reprogramming mostly relies on the extrinsic signaling of LIF and the intrinsic factor Oct4; LIF signaling is sufficient in reprogramming of epiblast cells, in which Oct4 is not expressed, into pluripotent ESCs $[32,33]$. Oct4 in a defined culture condition can reprogram somatic cells into pluripotent cells [34]. It has also been revealed that a reversible path from stem cells to differentiation in the germ cell lineage exists [35-37]. In addition, a few studies have demonstrated that a small fraction of mouse SSCs can be reprogrammed back to embryonic stem-like cells [5-9]. Based on these findings, we thought as in the reprogramming epiblast cells into ES-like cells [32,33], the LIF signaling might trigger the dedifferentiation of SSCs. In the present study, we found that SSCs can dedifferentiate back into PGCs and transdifferentiate into oocytelike cells when cultured in KO-DMEM medium containing $1 \%$ FBS, LIF plus $2 \mathrm{i}$ followed by DMEM/F12 medium supplemented with 15\% FBS, LIF, FSH, EGF, B27, and ITS. This observation is consistent with earlier findings concerning the reprogramming capability of the LIF signaling in the presence of $2 i[13,14]$ and further indicates the remarkable plasticity of SSCs in culture.

In our study, SSCs from 8 day-old testes were isolated and characterized as $\mathrm{GFRa}^{+} / \mathrm{PLZF}^{+}$but c-kit. C-kit is a key marker of PGCs, and lack of c-kit expression excluded the presence of PGCs in the isolated cells. Thus, the isolated SSCs were unipotent. We used SSCs from 8 day-old OG2 male mice carrying EGFP transgene under an Oct4 promoter to trace the dedifferentiation. In situ, we can only observe green cells in testes of OG2 mice before postnatal day 6 . We cultured SSCs from 8 days old testes, and found that $\sim 20 \%$ off total SSCs were induced to express EGFP within one week, thereby indicating the dedifferentiation of SSCs. Despite of the presence of EGFP green cells that indicated they were positive of Oct4, we did not observe any ESC-like colonies formed from these cells. Furthermore, we found that E-cadherin expression was absent in them and teratoma could not form them in nude mice, indicating they were not ESCs but more like PGCs. This was further confirmed by the expression of Blimp1 and Nanog. These cells continued to grow with increasing size, expressed Stella, Nobox, and GDF9, and demonstrated morphology resembling that of oocytes. More interestingly, at around day 25 in culture, we observed $\sim 2 \%$ of them developed into large cells with a polar body like MII oocytes. With our characterization of gene expression and morphology, we have clearly demonstrated that 


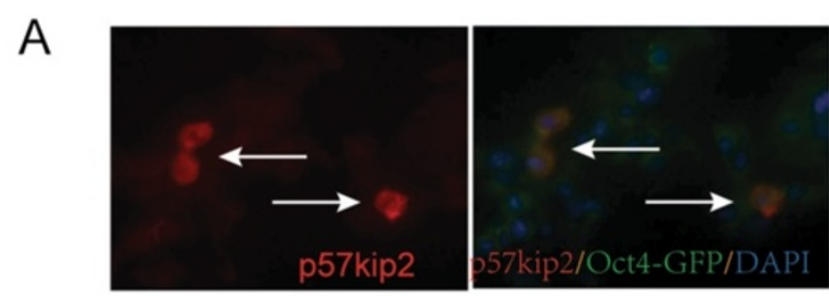

B SNRPN-DMR

SSC

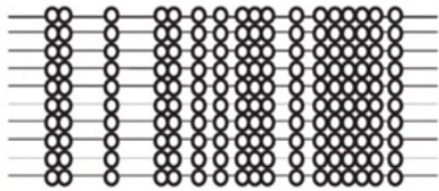

OG2-SSC

-Ooc

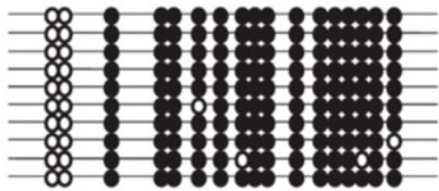

\section{Dik1-Gt12/Meg3 IG-DMR}

SSC
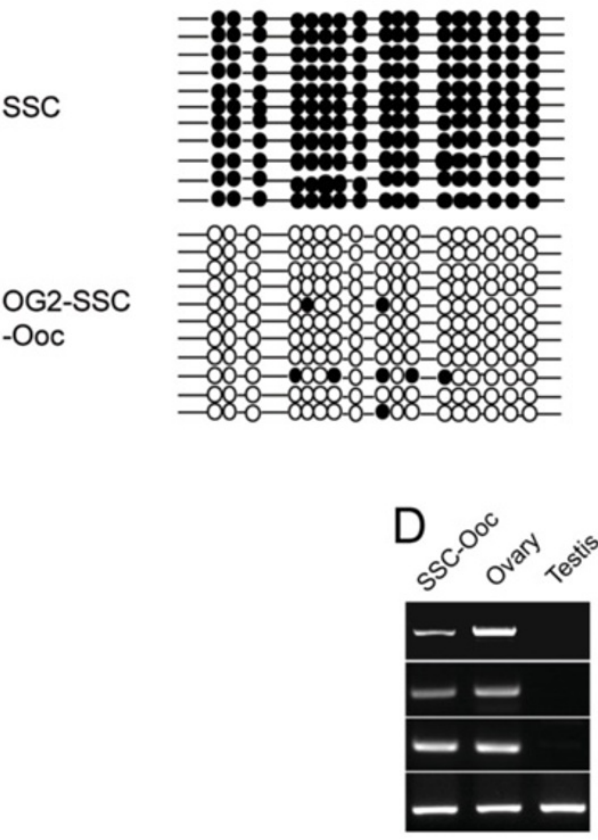

BMP15(X)
GDF9(11)
Usp9x(X)
GAPDH

IGF-H19-DMR
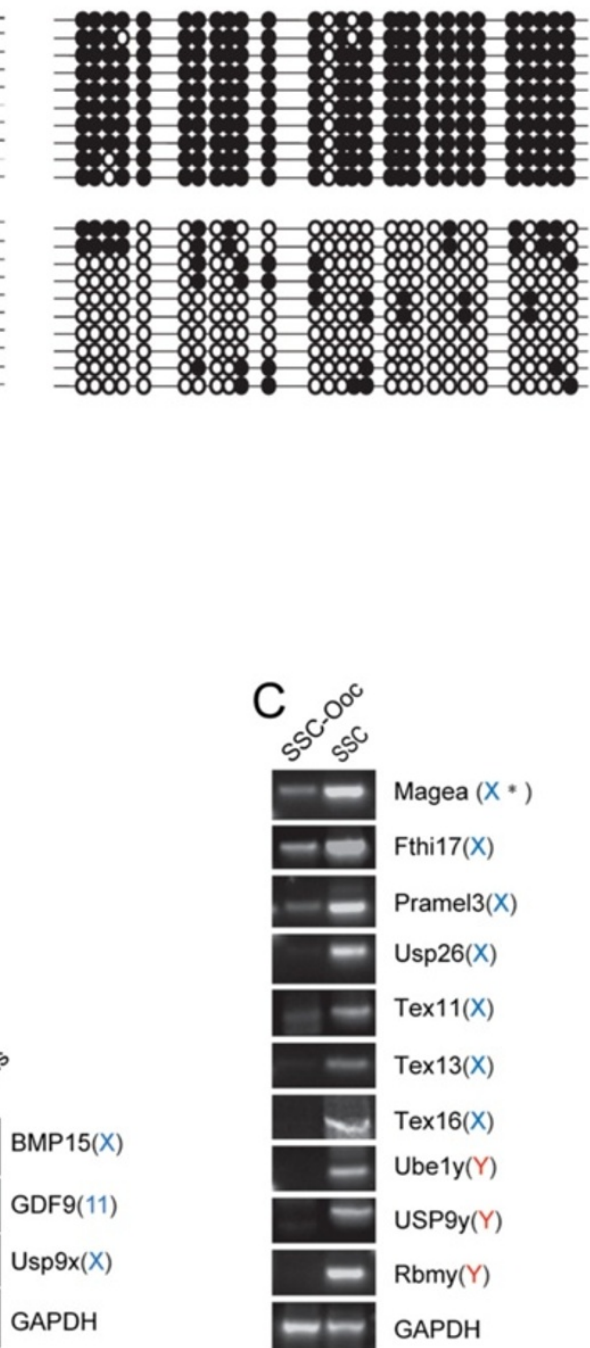

Figure 5 Analysis of Sex-specific imprint pattern and sex chromosome-linked gene activation. (A) Immunofluorescence of p57KIP2 in large cells derived from the OG2-SSC-Oct4/GFP cells (arrows). (B) Bisulfite genomic sequencing analyses of the DMR methylation status of parental origin-specific DNA methylation genes including Snrpn-DMR, Igf2-H19-DMR, and Dlk1-Meg3/GtI2-DMR; filled ovals indicate methylated CpGs, and open ovals indicate unmethylatedCpGs (SSCs carry hypermethylation of Igf2-H19-DMR and Dlk1-Meg3/Gt|2-DMR but hypomethylation of Snrpn-DMR; this pattern was reversed in the OG2-SSC-Oocs). (C) Examination of the expression of sex chromosome-linked genes in OG2-SSCOocs: RT-PCR analyses showing that 7 testis-specific X-linked genes were significantly down-regulated (Magea, Fthi17, and Pramel3) or turned off (Usp26, Tex11, Tex13, and Tex16), three Y-linked genes (Usp9y, Ube1y, and Rbmy) were not expressed; GAPDH was used as the RT-PCR control. (D)RT-PCR analyses showing that oocyte-specific genes including X-linked genes (Usp9x and Bmp15) and autosomal gene GDF9 were turned on in OG2-SSC-Oocs; GAPDH was used as the RT-PCR control. 
oocyte-like cells can be derived from SSCs via PGCs intermediates.

The imprinting patterns are established during gametogenesis with paternal imprints occurring during spermatogenesis and maternal imprints occurring during oogenesis. Defects in imprinting in gametes can give rise to severe problem in embryogenesis and predispose affected individuals to associated diseases after birth. To address if there were imprinting reversals in the conversion of SSCs into oocyte-like cells, we examined three key imprinting events including H19/Igf2, Snrpn, and Dik1-Gtl2/Meg3. It turned out that all three of them were switched to maternal status following the induction of oocyte-like cells from SSCs. Thus, in contrast to early report of germline-derived pluripotent stem (gPS) cells, which retain their original imprinting status [38], the reprogramming from SSCs to oocyte-like cells was accompanied by imprinting reversal. Therefore, SSCs possess both cellular and epigenetic plasticity and even give rise to oocyte-like cells in vitro in a manner similar to cases of sex reversal in vivo. Finally, we also demonstrated that a small number of SSC-Oocs were capable of being fertilized by sperm in vitro.

\section{Conclusions}

Our study has demonstrated that SSCs possess the potential to be reprogrammed into oocyte-like cells in culture. If culture conditions are further optimized, for example, using 3D culture system with ideal supportive cell feeder [39], to develop oocytes of high quality from SSCs, such a germ cell fate switch system should provide a useful in vitro model to study epigenetic regulation in oogenesis and sex reversal, furthering our understanding of the mechanisms that establish imprinting during gametogenesis.

\section{Methods}

\section{Ethics statement}

This study was conducted in compliance with the National Institutes of Health Guide for the Care and Use of Laboratory Animals with the approval (SYXK-20030026) of the Scientific Investigation Board of Shanghai Jiao Tong University School of Medicine, Shanghai, China. Mice were euthanized by $\mathrm{CO}_{2}$ inhalation to ameliorate any suffering throughout these experimental studies.

\section{Cell culture}

We used two enzymatic steps to isolate the adult ovarian germ cells from a 60-day old nursing BALB/c female mouse, whose male pups were used to isolate SSCs. The ovarian tissue was cut into small pieces followed by trypsin digestion for 5 minutes, then washed with DMEM/ F12 (GIBCO, Grand Island, NY, USA) once followed by treatment with $0.1 \%$ bovine testicular hyaluronidase (Sigma Aldrich, St Louis, MO, USA) for 20 minutes. Cells were dispersed by pipette and suspended in DMEM/F12 medium containing 1\% FBS (Biochrom AG, Berlin, Germany) and seeded onto 6 well plates. After 24 hours culture, the round cells, which were mostly on the top of plate-adhering cells, were collected by pipette and cultured in the DMEM/F12 medium containing 15\% FBS, 1,500 units/ml LIF (ESGRO, Chemicon, Billerica, MA, USA), $0.5 \mathrm{IU} / \mathrm{ml} \mathrm{FSH,} 10 \mathrm{ng} / \mathrm{ml}$ EGF (Invitrogen, Carlsbad, CA, USA), B27 (GIBCO), and ITS supplement (GIBCO).

SSCs were isolated from 8-day old OG2 mice using twostep enzymatic digestion followed by MACS (Miltenyi Biotech, BergischGladbach, Germany) using GFRa1 antibody, a goat anti-mouse antibody recognizing the $\mathrm{C}$-terminus of the GFRa1 receptor (Santa Cruz Biotechnology, Santa Cruz, CA, USA), with a 1:200 dilution. Isolated SSCs were cultured in gelatin-coated 6-well plates with KO-DMEM medium containing 1\% FBS, 1,500 units/ml LIF, and SU5402 (2 $\mu \mathrm{M}$, Calbiochem, La Jolla, CA, USA) plus CHIR99021(3 $\mu \mathrm{M}$, Axon Medchem, Groningen, Netherland)-2i, for one week; After GFP positive cells appeared, DMEM/F-12 medium containing 15\% FBS, 1,500 units/ml LIF, 100 microM 2-mercaptoethanol (GIBCO), FSH $(0.5 \mathrm{IU} / \mathrm{ml})$, EGF (10 ng/ml), B27, and ITS supplement was used for further culture.

\section{PCR, RT-PCR, and immunofluorescence}

For PCR and RT-PCR, we used the primers listed in Additional file 5: Table S1. E 3.0 Embryos were collected from pregnant mice for analysis of the expression of Trim43a and Hmgpi. For immunofluorescence, the following antibodies were used: GFRa1 antibody (goat polyclonal ,Santa Cruz Biotechnology); PLZF antibody (rabbit polyclonal, Abcam, Cambridge, MA, USA); Antip57 Kip2 (Cell Signaling, Inc. Danvers, MA, USA); Nobox antibody (rabbit polyclonal, Abcam); Stella antibody (rabbit polyclonal, Abcam); Mos antibody (rabbit polyclonal, Santa Cruz Biotechnology); Nanog antibody (rabbit polyclonal, Abcam); Gamma-tubulin antibody (mouse monoclonal, Sigma-Aldrich); We utilized specific markers for nuclear envelope (lamin B1) and nucleic acids (YoYo1) to demonstrate the presence of polar bodies in oocytes, Lamin B1 antibody(1:100, Rabbit polyclonal, Abcam), and YOYO-1 (Molecular Probes, YOYO $^{\circledR}$-1 Iodide $(491 / 509)$ ), diluted in phosphatebuffered saline to make $2.4 \mathrm{nM}$ to use.

\section{Intracytoplasmic sperm injection (ICSI)}

SSC-Oocs were rinsed thoroughly and kept in HepesCZB in original culture dish before injection. Adult OG2 mice were used as the donor for Oct4/GFP-carrying sperm. To retrieve sperm, seminiferous tubules were 
collected and put in Hepes-CZB. They were then cut into small pieces with a pair of fine scissors. A drop of the medium with tubule fragments was mixed with the same volume of Hepes-CZB containing 12\% (w: v) polyvinylpyrrolidone and pipetted vigorously to release spermatozoa. Sperm were collected and injected into SSC-Oocs. Injected oocytes were activated by $30-\mathrm{min}$ treatment with $\mathrm{Ca}^{2+}$-free $\mathrm{CZB}$ containing $5 \mathrm{mM} \mathrm{SrCl}_{2}$. Embryos were cultured in $\mathrm{KSOM}$ at $37^{\circ} \mathrm{C}$ in $5 \% \mathrm{CO}_{2}$.

\section{Bisulfite methylation analysis}

Genomic DNA was isolated from SSCs and SSC-Oocs. Bisulfite conversion was performed on a thermocycler using the QiagenEpiTect Kit (Qiagen, Hilden, Germany) according to manufacturer's instructions, with two additional cycles $\left(5 \mathrm{~min}\right.$ at $99^{\circ} \mathrm{C}$ and $3 \mathrm{~h}$ at $60^{\circ} \mathrm{C}$ ) at the end. Converted DNA was eluted in $40 \mu \mathrm{l}$ of elution buffer, and a 5- $\mu$ DNA sample was then amplified with the following primer sets: Snrpn, AATTTGTGTGATGTTTG TAATTATTTGG and ATAAAATACACTTTCACTACT AAAA TCCACAA; Igf2-H19-DMR, GGAATATTTGT GTTTTTGGAGGG and TTAAACCCCAACCTCTAC TTTT ATAAC; Dlk1-Meg3/Gtl2-DMR, GGTTTGG TATATATGGATGT ATTGTAATATAGG and ATAAAA CACCAAATCTATACCAAAATATACC. PCR was performed in $25-\mu \mathrm{l}$ reactions using 2.5 units of ExTaq under the following conditions (38 cycles): 1 ) $96^{\circ} \mathrm{C}$ for $15 \mathrm{sec}-$ onds, $60^{\circ} \mathrm{C}$ for 30 seconds, and $72^{\circ} \mathrm{C}$ for 30 seconds for Snrpn; 2) $96^{\circ} \mathrm{C}$ for 15 seconds (hot start), $55^{\circ} \mathrm{C}$ for 30 seconds, and $72^{\circ} \mathrm{C}$ for 1 minute for Igf2-H19-DMR and Dlk1-Meg3/Gtl2-DMR. The amplified fragments were cloned into the pMD19-T vector (TaKaRa Biotech Co., Ltd) and then sequenced.

\section{Statistics}

All experiments were performed 4 times, and data were expressed as means \pm SE and analyzed by one-way ANOVA analysis. A value of $P<0.05$ was considered significant.

\section{Additional files}

Additional file 1: Figures S1. (A) Culture of ovarian germ cells from an adult BABL/C female in DMEM/F12 + 15\% FBS + LIF showing that GCNA positive cells (red, identified by immunofluorescent staining with GCNA antibody provided from George Enders, University of Kansas Medical Center). (B) RT-PCR analyses showing the expression of GDF-9, Nobox, and Oogenesin in oocytes and SSC-derived Oocytes.

Additional file 2: Figure S2. (A) Immunofluorescence of SCP3 showing a meiotic SSC-derived oocyte. (B) Giemsa staining demonstrates a SSCderived haploid oocyte.

Additional file 3: Figures S3. Time-course staining of the markers of primordial germ cells (PGCs) and oocytes. BALB/C SSCs were isolated and cultured in KO-DMEM for one week, then cultured in DMEM/F12 medium and subject to immunofluorescence staining at different times with antibodies against primordial germ cells (PGCS) and oocyte markers, including Nanos2, Nanos3, Nanog, Blimp1, and Nobox.
Additional file 4: Table S2. In SSC-Oocs, X-and Y-linked testis specific genes were turned off, $X$-linked ovary specific genes were turned on. GDF9, an oocyte specific gene, was turned on too.

Additional file 5: Table S1. PCR Primers.

\section{Competing interests}

The authors declare that they have no competing interest.

\section{Author's contributions}

PJ helped the cell culture, JC did Bisulfite methylation analysis, DZ and LM prepared animals and isolated germ cells, LW and ZY carried out most experiments, MD and LF designed the overall study, ZY and LF prepared the manuscript. All authors read and approved the final manuscript.

\section{Acknowledgments}

We thank Jinsong Li of Institute of Biochemistry and Cell Biology/Shanghai Institutes for Biological Sciences/Chinese Academy of Sciences for providing OG2 mice. We thank Haifan Lin of Yale University for their critical and useful comments on the manuscript.

This work was partially supported by grants from the National Key Basic Research and Development Program of China (2009CB941103) and from the National Natural Science Foundation of China (30870956) awarded to Dr. Feng; from the National Natural Science Foundation of China (81000049) awarded to Dr. Yu; and from the 211 program of Shanghai Jiao Tong University School of Medicine and Shanghai Leading Academic Discipline Project (S30201).

\section{Author details}

${ }^{1}$ Institute of Medical Sciences, Shanghai JiaoTong University School of Medicine, 280 Chongqing S. Road, Shanghai 200025, China. ${ }^{2}$ Institute of Health Sciences, Shanghai Institutes for Biological Sciences, Chinese Academy of Sciences, Shanghai, China. ${ }^{3}$ Department of Urology, General Hospital of Ningxia Medical University, Yinchuan, Ningxia, China.

${ }^{4}$ Department of Biochemistry and Molecular \& Cellular Biology, Georgetown University Medical Center, Washington, DC 20057, USA.

Received: 13 July 2012 Accepted: 16 July 2012

Published: 6 August 2012

\section{References}

1. Adams IR, McLaren A: Sexually dimorphic development of mouse primordial germ cells: switching from oogenesis to spermatogenesis. Development 2002, 129(5):1155-64.

2. McLaren A: Sex chimaerism and germ cell distribution in a series of chimaeric mice. J Embryol Exp Morphol 1975, 33(1):205-16.

3. McLaren A: The fate of germ cells in the testis of fetal Sex-reversed mice. J Reprod Fertil 1981, 61(2):461-7.

4. Koopman P, Munsterberg A, Capel B, Vivian N, Lovell-Badge R: Expression of a candidate sex-determining gene during mouse testis differentiation. Nature 1990, 348(6300):450-2.

5. Conrad S, Renninger M, Hennenlotter J, Wiesner T, Just L, Bonin M, Aicher W, Buhring HJ, Mattheus U, Mack A, Wagner HJ, Minger S, Matzkies M, Reppel M, Hescheler J, Sievert KD, Stenzl A, Skutella T: Generation of pluripotent stem cells from adult human testis. Nature 2008, 456(7220):344-9.

6. Golestaneh N, Kokkinaki M, Pant D, Jiang J, DeStefano D, Fernandez-Bueno C, Rone JD, Haddad BR, Gallicano Gl, Dym M: Pluripotent stem cells derived from adult human testes. Stem Cells Dev 2009, 18(8):1115-26.

7. Guan K, Nayernia K, Maier LS, Wagner S, Dressel R, Lee JH, Nolte J, Wolf F, Li M, Engel W, Hasenfuss G: Pluripotency of spermatogonial stem cells from adult mouse testis. Nature 2006, 440(7088):1199-203.

8. Kanatsu-Shinohara M, Inoue K, Lee J, Yoshimoto M, Ogonuki N, Miki H, Baba S, Kato T, Kazuki Y, Toyokuni S, Toyoshima M, Niwa O, Oshimura M, Heike T, Nakahata T, Ishino F, Ogura A, Shinohara T: Generation of pluripotent stem cells from neonatal mouse testis. Cell 2004, 119(7):1001-12.

9. Kossack N, Meneses J, Shefi S, Nguyen HN, Chavez S, Nicholas C, Gromoll J, Turek PJ, Reijo-Pera RA: Isolation and characterization of pluripotent human spermatogonial stem cell-derived cells. Stem Cells 2009, 27(1):138-49. 
10. Hubner K, Fuhrmann G, Christenson LK, Kehler J, Reinbold R, De La Fuente R, Wood J, Strauss JF 3rd, Boiani M, Scholer HR: Derivation of oocytes from mouse embryonic stem cells. Science 2003, 300(5623):1251-6.

11. Meng $X$, Lindahl M, Hyvonen ME, Parvinen M, de Rooij DG, Hess MW, Raatikainen-Ahokas A, Sainio K, Rauvala H, Lakso M, Pichel JG, Westphal H, Saarma M, Sariola H: Regulation of cell fate decision of undifferentiated spermatogonia by GDNF. Science 2000, 287(5457):1489-93.

12. Hofmann MC, Braydich-Stolle L, Dym M: Isolation of male germ-line stem cells; influence of GDNF. Dev Biol 2005, 279(1):114-24.

13. Li P, Tong C, Mehrian-Shai R, Jia L, Wu N, Yan Y, Maxson RE, Schulze EN, Song H, Hsieh CL, Pera MF, Ying QL: Germline competent embryonic stem cells derived from rat blastocysts. Cell 2008, 135(7):1299-310.

14. Li W, Wei W, Zhu S, Zhu J, Shi Y, Lin T, Hao E, Hayek A, Deng H, Ding S: Generation of rat and human induced pluripotent stem cells by combining genetic reprogramming and chemical inhibitors. Cell Stem Cell 2009, 4(1):16-9.

15. Dong J, Albertini DF, Nishimori K, Kumar TR, Lu N, Matzuk MM: Growth differentiation factor- 9 is required during early ovarian folliculogenesis. Nature 1996, 383(6600):531-5.

16. Rajkovic A, Pangas SA, Ballow D, Suzumori N, Matzuk MM: NOBOX deficiency disrupts early folliculogenesis and oocyte-specific gene expression. Science 2004, 305(5687):1157-9.

17. Minami N, Aizawa A, Ihara R, Miyamoto M, Ohashi A, Imai H: Oogenesin is a novel mouse protein expressed in oocytes and early cleavage-stage embryos. Biol Reprod 2003, 69(5):1736-42.

18. De La Fuente R: Chromatin modifications in the germinal vesicle (GV) of mammalian oocytes. Dev Biol 2006, 292(1):1-12.

19. Tanaka M, Hennebold JD, Macfarlane J, Adashi EY: A mammalian oocytespecific linker histone gene $\mathrm{H} 1 \mathrm{Oo}$ : homology with the genes for the oocyte-specific cleavage stage histone (cs-H1) of sea urchin and the B4/ $\mathrm{H} 1 \mathrm{M}$ histone of the frog. Development 2001, 128(5):655-64.

20. Stanghellini I, Falco G, Lee SL, Monti M, Ko MS: Trim43a, Trim43b, and Trim43c: Novel mouse genes expressed specifically in mouse preimplantation embryos. Gene Expr Patterns 2009, 9(8):595-602

21. Yamada M, Hamatani T, Akutsu H, Chikazawa N, Kuji N, Yoshimura Y Umezawa A: Involvement of a novel preimplantation-specific gene encoding the high mobility group box protein Hmgpi in early embryonic development. Hum Mol Genet 2010, 19(3):480-93.

22. Saitou M, Barton SC, Surani MA: A molecular programme for the specification of germ cell fate in mice. Nature 2002, 418(6895):293-300.

23. Fujiwara Y, Komiya T, Kawabata H, Sato M, Fujimoto H, Furusawa M, Noce T: Isolation of a DEAD-family protein gene that encodes a murine homolog of Drosophila vasa and its specific expression in germ cell lineage. Proc Natl Acad Sci U S A 1994, 91(25):12258-62.

24. Tsuda M, Sasaoka Y, Kiso M, Abe K, Haraguchi S, Kobayashi S, Saga Y: Conserved role of nanos proteins in germ cell development. Science 2003, 301(5637):1239-41.

25. Chambers I, Silva J, Colby D, Nichols J, Nijmeijer B, Robertson M, Vrana J, Jones K, Grotewold L, Smith A: Nanog safeguards pluripotency and mediates germline development. Nature 2007, 450(7173):1230-4

26. Ohinata Y, Payer B, O'Carroll D, Ancelin K, Ono Y, Sano M, Barton SC, Obukhanych T, Nussenzweig M, Tarakhovsky A, Saitou M, Surani MA: Blimp1 is a critical determinant of the germ cell lineage in mice. Nature 2005, 436(7048):207-13.

27. Sada A, Suzuki A, Suzuki H, Saga Y: The RNA-binding protein NANOS2 is required to maintain murine spermatogonial stem cells. Science 2009, 325(5946):1394-8.

28. Hatada I, Mukai T: Genomic imprinting of p57KIP2, a cyclin-dependent kinase inhibitor, in mouse. Nat Genet 1995, 11(2):204-6.

29. Dube JL, Wang P, Elvin J, Lyons KM, Celeste AJ, Matzuk MM: The bone morphogenetic protein 15 gene is X-linked and expressed in oocytes. Mol Endocrinol 1998, 12(12):1809-17.

30. Wang PJ, McCarrey JR, Yang F, Page DC: An abundance of X-linked genes expressed in spermatogonia. Nat Genet 2001, 27(4):422-6.

31. Noma T, Kanai Y, Kanai-Azuma M, Ishii M, Fujisawa M, Kurohmaru M, Kawakami H, Wood SA, Hayashi Y: Stage- and sex-dependent expressions of Usp9x, an X-linked mouse ortholog of Drosophila Fat facets, during gonadal development and oogenesis in mice. Mech Dev 2002, 119 (Suppl 1):S91-5.
32. Bao S, Tang F, Li X, Hayashi K, Gillich A, Lao K, Surani MA: Epigenetic reversion of post-implantation epiblast to pluripotent embryonic stem cells. Nature 2009, 461(7268):1292-5.

33. Buecker $\mathrm{C}$, Chen HH, Polo JM, Daheron L, Bu L, Barakat TS, Okwieka P, Porter A, Gribnau J, Hochedlinger K, Geijsen N: A murine ESC-like state facilitates transgenesis and homologous recombination in human pluripotent stem cells. Cell Stem Cell 2010, 6(6):535-46.

34. Kim JB, Greber B, Arauzo-Bravo MJ, Meyer J, Park Kl, Zaehres H, Scholer HR: Direct reprogramming of human neural stem cells by OCT4. Nature 2009, 461(7264):649-3.

35. Brawley C, Matunis E: Regeneration of male germline stem cells by spermatogonial dedifferentiation in vivo. Science 2004, 304(5675):1331-4

36. Kai T, Spradling A: Differentiating germ cells can revert into functional stem cells in Drosophila melanogaster ovaries. Nature 2004, 428(6982):564-9.

37. Nakagawa T, Sharma M, Nabeshima Y, Braun RE, Yoshida S: Functional hierarchy and reversibility within the murine spermatogenic stem cell compartment. Science 2010, 328(5974):62-7

38. Ko K, Tapia N, Wu G, Kim JB, Bravo MJ, Sasse P, Glaser T, Ruau D, Han DW, Greber B, Hausdorfer K, Sebastiano V, Stehling M, Fleischmann BK, Brustle O, Zenke M, Scholer HR: Induction of pluripotency in adult unipotent germline stem cells. Cell Stem Cell 2009, 5(1):87-96.

39. Eppig JJ, O'Brien MJ: Development in vitro of mouse oocytes from primordial follicles. Biol Reprod 1996, 54(1):197-207.

doi:10.1186/2045-3701-2-27

Cite this article as: Wang et al:: Oocyte-like cells induced from mouse spermatogonial stem cells. Cell \& Bioscience 2012 2:27.

\section{Submit your next manuscript to BioMed Central and take full advantage of:}

- Convenient online submission

- Thorough peer review

- No space constraints or color figure charges

- Immediate publication on acceptance

- Inclusion in PubMed, CAS, Scopus and Google Scholar

- Research which is freely available for redistribution

Submit your manuscript at www.biomedcentral.com/submit
C Biomed Central 